\title{
Developing Effective Electrodes for Supercapacitors by Grafting of Trihydroxybenzene onto Activated Carbons
}

\author{
David Malka ${ }^{a}$, Shaul Bublil ${ }^{a}$, Ran Attias ${ }^{a}$, Michal Weitmana, Reut Cohen ${ }^{a}$, Yuval Elias ${ }^{a}$, Yosef Gofer ${ }^{a}$, \\ Thierry Brousse ${ }^{\mathrm{b}, \mathrm{c} *}$, and Doron Aurbach ${ }^{\mathrm{a} *}$ \\ a Department of Chemistry, Bar-Ilan University, Ramat Gan 5290002, Israel \\ ${ }^{\mathrm{b}}$ Institut des Matériaux Jean Rouxel (IMN), Université de Nantes, CNRS, 44322 Nantes Cedex 3, France \\ c Réseau sur le Stockage Electrochimique de l’Energie (RS2E), FR CNRS 3459, France \\ * Corresponding author. Email: aurbach@mail.biu.ac.il
}

Keywords: Supercapacitors, Grafting, Activated carbon electrodes, Diazonium chemistry, Oxygen rich aromatic amines, Trihydroxybenzene

\begin{abstract}
The specific capacity of activated carbon electrodes for supercapacitors may be enhanced with additional faradaic redox reactions by grafting of electroactive aromatic molecules with heteroatoms that act as redox centers. Such enrichment was demonstrated recently with anthraquinone and catechol using diazonium chemistry. Here, trihydroxybenzene, which has obvious advantages, was successfully grafted, yielding a mass enrichment of $25 \%$. Electrochemical characterization in acidic aqueous solution after in situ methoxy deprotection demonstrated an initial specific capacity of $65 \mathrm{mAh} / \mathrm{g}$, which faded only slightly to $55 \mathrm{mAh} / \mathrm{g}$ after about 2000 cycles and remained stable for over 4500 cycles.
\end{abstract}

\section{Introduction}

Most important electrochemical energy storage devices can be divided into two main categories according to the location of the electrochemical reactions - batteries and electrochemical capacitors $(E C s)^{1}$. Batteries utilize the whole bulk of the electrodes, leading to high energy density, albeit with some penalty associated with low power density due to kinetic limitations and a limited cycle life. In ECs, the electrochemical reactions take place at the surface of porous electrodes, leading to lower energy density, but with much lower kinetic limitations, and hence appreciably higher attainable power densities. ${ }^{2}$ Li-ion batteries (LIBs) were developed extensively during the last three decades ${ }^{3}$ and are at the core of the electric-mobility revolution, lowering the world dependence on fossil fuels and internal combustion engines (ICEs).

ECs hold important characteristics, which are absent in LIBs such as very prolonged cycle life, very highpower density, and excellent energy turnover per cycle (very low voltage hysteresis between charge and discharge processes). Therefore, such devices may be very useful for a variety of applications related to energy storage and conversion. Unfortunately, mainly due to insufficient energy density, ECs received less attention, and were not developed at the same scale as LIBs. However, despite their intrinsic lower energy density, ECs complement batteries owing to their fast-charging capability, inherent safety, low ecological footprint (raw material over device lifetime), and relatively low cost from manufacturing to disposal.

The possibility to increase the energy density of super capacitors yet reserve their unique properties like high-rate capability, Excellent safety features and impressive durability is very attractive and important. 


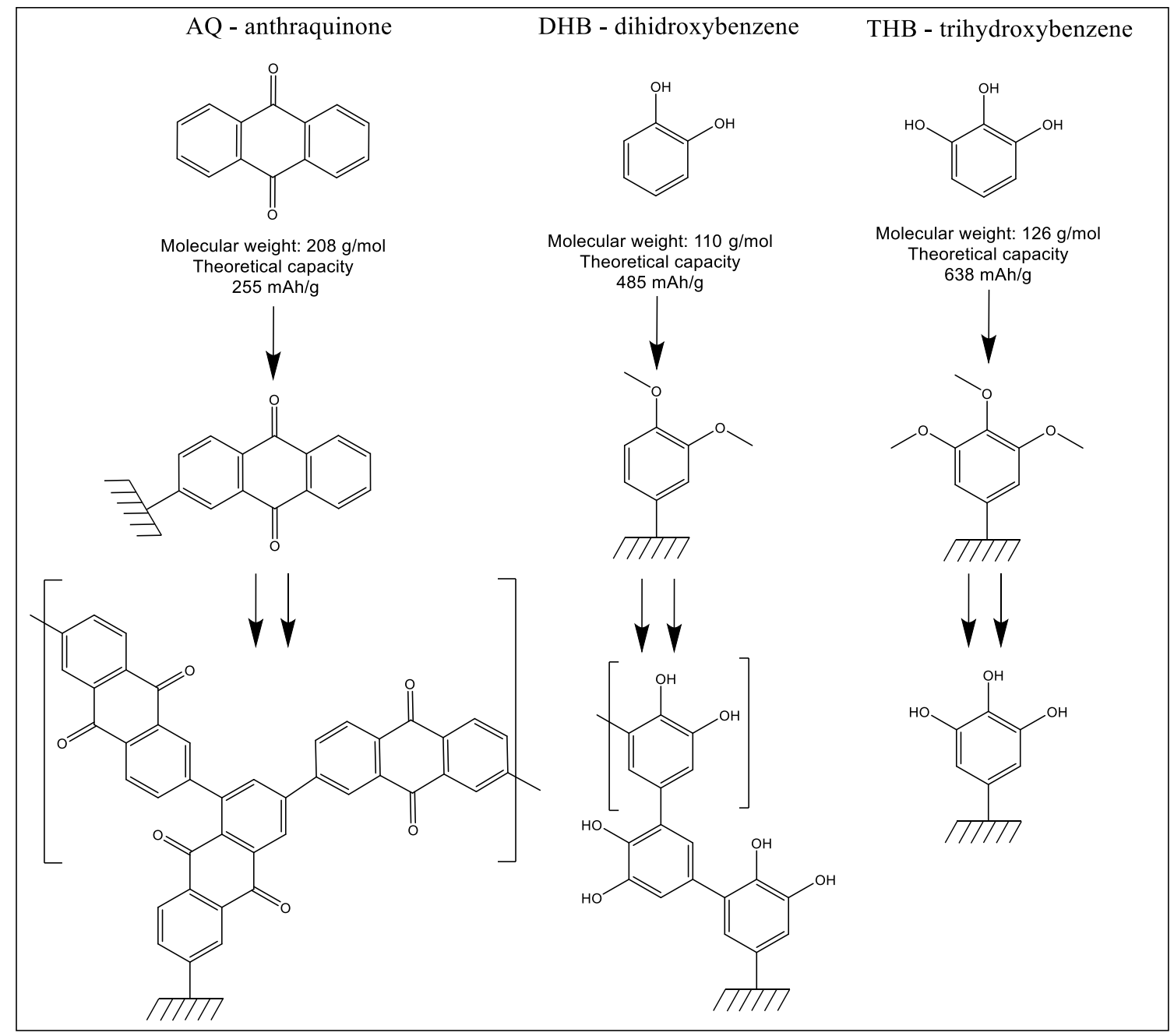

Figure 1: Grafting of electroactive molecules onto active carbon surface: the molecular weight and theoretical capacity are indicated, along with the dynamic formation of multilayer clusters on the porous surface. Branching on grafted molecules is more likely for AQ compared to DHB and is unlikely for THB.

Carbon based electrodes for electric double layer capacitors (EDLC) have intrinsic limitations which are related to the electrode/electrolyte solution interactions. The specific surface area and pore size distribution of a given carbon electrode rule the adsorption of ions from the electrolyte solution. However, the composition of the electrolyte solution also plays a decisive role in the adsorption and electro-sorption processes. The nature of the solvent molecules, the effective anion and cation size, and their mobility and concentration dominate their specific interaction with the carbon surface and determine the effectiveness of the porous structure: to what extent is the porous surface area accessible for ion adsorption? The effective surface area of porous electrodes for ion adsorption obviously depends on the applied potential. Higher potentials force more ions to enter into small pores despite steric limitations (e.g., forced desolvation that makes them effectively smaller). However, this in turn may lead to undesirable stress, which is detrimental to the electrode stability.

Another important optimization relates to the nature of the electrolyte solution. The specific capacity of many types of activated carbon electrodes may be higher by a factor of two in aqueous compared to nonaqueous solutions. Their rate capability may also be an order of magnitude faster. However, non-aqueous solutions allow much higher charging voltages, by a factor of 2.5 compared to aqueous systems, resulting in much higher energy density. For symmetric supercapacitors, the energy density is given by $C E^{2} / 8$, where $C$ is the specific capacitance of the electrode in $\mathrm{F} / \mathrm{g}$ (assuming negligible dependence on applied potential), and $E$ is the maximal applied potential (i.e., the charging potential). However, in non-aqueous solutions, 
supercapacitors might lose their high-power density, their main advantage. Electrode design clearly needs to be carefully matches with electrolyte solution formulation.

Another point for consideration is the electrolyte solution concentration. Carbon electrodes usually exhibit a larger specific capacitance in more concentrated aqueous solutions. In highly concentrated solutions, the applied potential can increase from 1 to $1.5 \mathrm{~V}$, hence using highly concentrated aqueous solutions may pronouncedly increase the energy density of supercapacitors. However, there might be an adverse effect on the conductivity, whereby the resistance of the solution is increased, resulting in hysteresis between the charge and discharge processes due to development of overpotentials.

The advantage of using carbon-based electrodes can be challenged by replacing them with other materials such as metal oxides, metal nitrides, or MXenes, which frequently act as pseudocapacitive electrodes, at the expense of recycling ability, cost, and complex hybrid design. Another approach, which avoids carbon replacement, is to increase the capacity of carbon electrodes by grafting electroactive moieties on their surface. In such a design, the electrode can benefit from additional capacity due to the grafted redox molecules while maintaining its intrinsic double layer capacitance, as demonstrated by Pognon et al. ${ }^{4}$ Diazonium chemistry is a powerful tool for modifying carbonaceous materials ${ }^{5}$ due to the simple one pot reaction, which requires no particular workup or purification stages. Grafting through diazonium chemistry was developed originally for the pigment industry ${ }^{6,7}$ and became the leading approach for carbon surface modifications. Anthraquinone ${ }^{8-12}(\mathrm{AQ})$ and catechol ${ }^{13-16}$ (dihydroxybenzene - DHB) were both reported extensively as redox active molecules for modification of activated carbons.

Here, we consider grafting of an alternative molecule, trihydroxybenzene (THB), which, to the best of our knowledge, was not previously proposed for modification of activated carbons. As shown in figure 1, the grafting process is continuous, and electroactive molecules from the solution are steadily anchored onto the surface of the carbon. The molecular weight of the grafted molecule and the number of electrons involved in the redox reaction determine the theoretical capacity. From a practical point of view, the available capacity depends on the mass and electroactivity of the grafted electroactive molecules, both in terms of capacity and redox potentials. Thus, branched molecules (Fig. 1) are undesirable, as they increase the total mass of the electrode without contributing to charge storage. AQ and DHB both have two electrons available for redox reactions, with a molecular weight and theoretical capacity of $208 \mathrm{~g} / \mathrm{mol}$ and $255 \mathrm{mAh} / \mathrm{g}$ and $110 \mathrm{~g} / \mathrm{mol}$ and $485 \mathrm{mAh} / \mathrm{g}$, respectively. THB, with an intermediate molecular weight of $126 \mathrm{~g} / \mathrm{mol}$ and three available electrons, has an impressive theoretical capacity of $638 \mathrm{mAh} / \mathrm{g}$, which makes it an interesting candidate for improving carbon-based electrodes.

The basic condition for grafting molecules is the presence of an aromatic amine. The vast number of possible molecular combinations deserve a discussion on form and function, as the grafted molecules are designed to store energy through electronic transitions empowered by resonance structure tautomerism and allylic system transformations. These include keto-enol, keto-imine, and keto-nitro tautomerism and carbocation stabilization of allylic radicals by overlapping $\pi$ orbitals from the conjugated system. These mechanisms are akin to activated carbons as well as to a great variety of heteroatoms in conjugated systems. The redox reaction should be reversible within the desired potential window. As illustrated in figure 2, the grafting mechanism progresses in two steps. The aromatic amine first reacts with a nitrite group (diazotation) to form an unstable diazonium derivative, which spontaneously breaks, releasing nitrogen gas and forming a reactive radical derivative. The grafted radical is then reduced by the conjugated double bonds at the activated carbon surface, so that the grafted molecule is attached to the surface of the carbon and joins the conjugated system. Alternatively, the diazonium derivative can bond with the surface via a diazo bridge but to a minor extend compared to the previous reaction scheme.

Another important aspect is the loading capability, the number of molecules eventually grafted. While the carbon matrix provides electrons to the diazonium derivative there is competition between direct grafting to the carbon surface, branching from grafted molecules on the surface and branching of molecules in 
solution. $A Q$ is a bulky molecule compared to $\mathrm{DHB}$ and $\mathrm{THB}$, and the regrafting process might lead to bulky clusters growing rapidly and blocking the smaller micropores, as it was earlier demonstrated by Pognon et al. ${ }^{9}$ Figure 1 schematically shows grafting with three layers of molecules. The structure of the DHB cluster is much more compact compared with that of the $A Q$ cluster, while for THB, a monolayer, essentially, of grafted molecules is expected across the carbon surface due to steric hindrance. Although ortho position of the pristine amine is free, it is available for dimer coupling only in solution when already bound to the carbon surface. Ortho positions allow only coupling in parallel to the surface which is very rare and cannot propagate further because of steric hindrance. Eventually, the loading of the grafted molecules is determined by a series of conditions, from the structure of the molecule and the stability of the diazonium salt or radical to the available sites for grafting. While surface coverage proceeds, the number of available sites decreases, either for grafting at the carbon surface, or for branching to an already grafted molecule. another important aspect that needs to be considered is whether the grafted molecule is readily available for redox reaction, like $A Q$, or requires a step of activation or deprotection, like THB. Recently ${ }^{8,15}$, carbon mass enrichment of $25 \%$ by weight was achieved for both AQ and DHB. Here, we sought to achieve a similar level of enrichment for THB, which would clearly present an advantage, as shown in figure 1.

\section{Experimental section}

\section{Materials and methods}

Activated carbon cloth of Kynol ${ }^{\mathrm{TM}}$ type 507-20 was purchased from Kynol Europa GmbH (Germany). 3,4,5trimethoxyaniline (TMA, 97\%) was purchased from Aldrich (Israel), and tert-butyl nitrite (90\% solution in t-butanol) was acquired from Alfa Aesar (USA). Unless otherwise stated, all other reagents were obtained from Aldrich, and used without further purification.

\section{Carbon modification}

As illustrated in figure 2, $100 \mathrm{mg}$ of 3,4,5-trimethoxyaniline (TMA, $0.546 \mathrm{mmol}$ ) were dissolved in $100 \mathrm{ml}$ of acetonitrile until complete dissolution of the amine derivative. Three equivalents of tert-butyl nitrite were added $\left(0.240 \mathrm{ml}\right.$ of a $90 \%$ solution), followed by a $100 \mathrm{mg}$ piece of $\mathrm{Kynol}^{\mathrm{TM}}$ carbon cloth. The mixture was stirred for 24 hours, and the modified piece of cloth was filtered and washed with aliquots of dimethylformamide, acetonitrile, acetone, and methanol and dried at $60^{\circ} \mathrm{C}$ under vacuum, yielding 125 $\mathrm{mg}$ of modified carbon ( $25 \mathrm{wt} \%$ addition of grafted molecule).

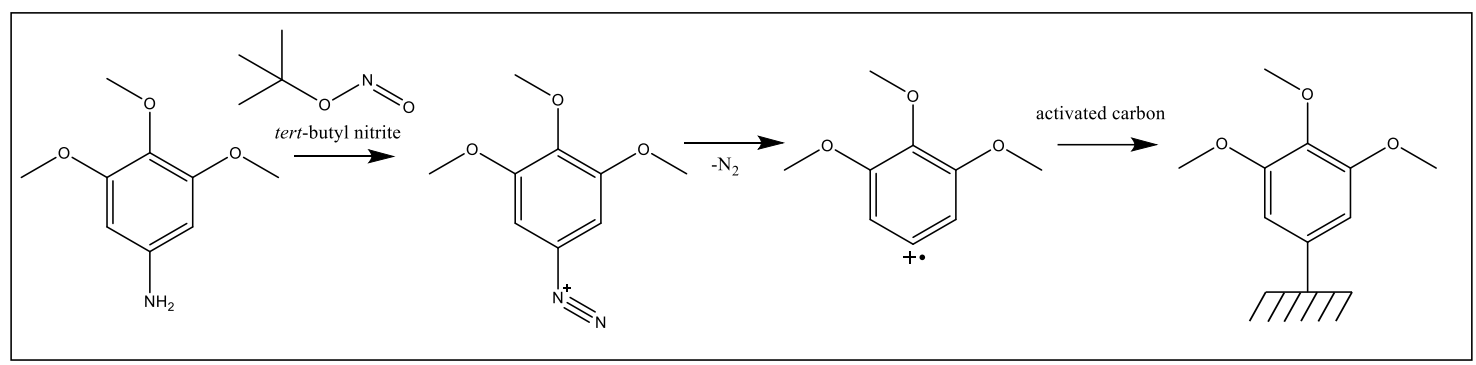

Figure 2: Grafting of THB using diazonium chemistry.

\section{Surface analysis}

Surface area measurements were carried out in a Quantachrome NOVA 3200e surface area and pore size analyzer with nitrogen as an adsorbent. Thermogravimetric analysis (TGA) was conducted using a TGAGC-MS (EI/Cl) Clarus 680/Clarus SQ 8C instrument (Perkin Elmer, USA) under nitrogen atmosphere to evaluate the thermal stability of the grafted species. Surface elemental and chemical state analysis was performed by X-ray photoelectron spectroscopy (XPS). The XPS study was carried with a Thermo Scientific Nexsa spectrometer with a monochromated Al K $\alpha$ source. Survey spectra were recorded at PE of $200 \mathrm{eV}$, while high resolution spectra were acquired at $50 \mathrm{eV}$. In situ charge neutralization was used, and each set of measurements was recalibrated versus $\mathrm{C} 1 \mathrm{~s}$ at $284.8 \mathrm{eV}$. due to the conducting nature of the samples, 
only slight charging was observed, in the ballpark of a few tens of meV. Quantification was done after "smart" baseline correction, using relative sensitivity factors (RSF) provided by the Nexsa system. Peak fiittings were carried out with Gaussian-Lorentian 70:30 peaks and reasoneable full width at half maximum (FWHM) values.

\section{Electrochemical measurements}

All electrochemical measurements were carried out in Swagelok three electrode T cells. Electrodes were made of PTFE cylinder assembled with two $4 \mathrm{~mm}$ glassy carbon rods, serving as both current collectors and terminals. Working electrodes were punched from the modified carbon cloth $6 \mathrm{~mm}$ in diameter with an average weight of $7 \mathrm{mg}\left(\approx 25 \mathrm{mg} / \mathrm{cm}^{2}\right)$, resembling real life electrodes found in commercial devices. Counter electrodes of $2 \times 12 \mathrm{~mm}$ in diameter were punched from unmodified carbon cloth with average weight of $46 \mathrm{mg}$ (double disks of $12 \mathrm{~mm}$ ), resulting in an electrode mass loading of about $40 \mathrm{mg} / \mathrm{cm}^{2}$. NKK cellulose paper was used as a separator. Electrodes were immersed in distilled water and slightly swirled under vacuum to allow good wetting, followed by soaking in $2 \mathrm{M} \mathrm{H}_{2} \mathrm{SO}_{4}$ solution to allow the osmotic pressure to bring the solution into the pores within the entire electrode mass. A saturated calomel electrode (SCE) was used as reference electrode.

\section{Results and discussion}

Thermogravimetric analysis (TGA) performed under nitrogen at a temperature rate of $10 \mathrm{~K} / \mathrm{min}$ for $\mathrm{AQ}$ and di/trimethoxybenzene (DMB/TMB), the methoxy protected derivatives of DHB/THB, vs. pristine activated carbon are shown in figure 3. Different patterns of weight loss are observed for each molecule as the temperature rises. The factors that influence and shape the weight loss pattern are varied, including the chemical nature of the weakly adsorbed molecule, the physical structure of the branched cluster (number of atoms derived from the root bond), the distance of the oxygen atoms from the root bond, and the total $\mathrm{C} / \mathrm{O}$ ratio, which should promote rapid degradation pathways. $\mathrm{AQ}$ which has the longest distance between the root bond and an oxygen atom shows a relatively minimal weight loss of about $10 \%$ up to $450{ }^{\circ} \mathrm{C}$ due to much lower ratio of oxygen to carbon $(C / O=7)$ compared to both $\mathrm{DMB}$ and TMB. $\mathrm{DMB}$ shows a massive weight loss of about $50 \%$ in this temperature range, related to cluster formation, empowered by the high ratio of oxygen to carbon $(\mathrm{C} / \mathrm{O}=3)$, which creates decomposition centers, while TMB exhibits a moderate weight loss of $20 \%$, mainly due to the compact grafted structure, despite the high oxygen to carbon ratio $(\mathrm{C} / \mathrm{O}=2)$. 


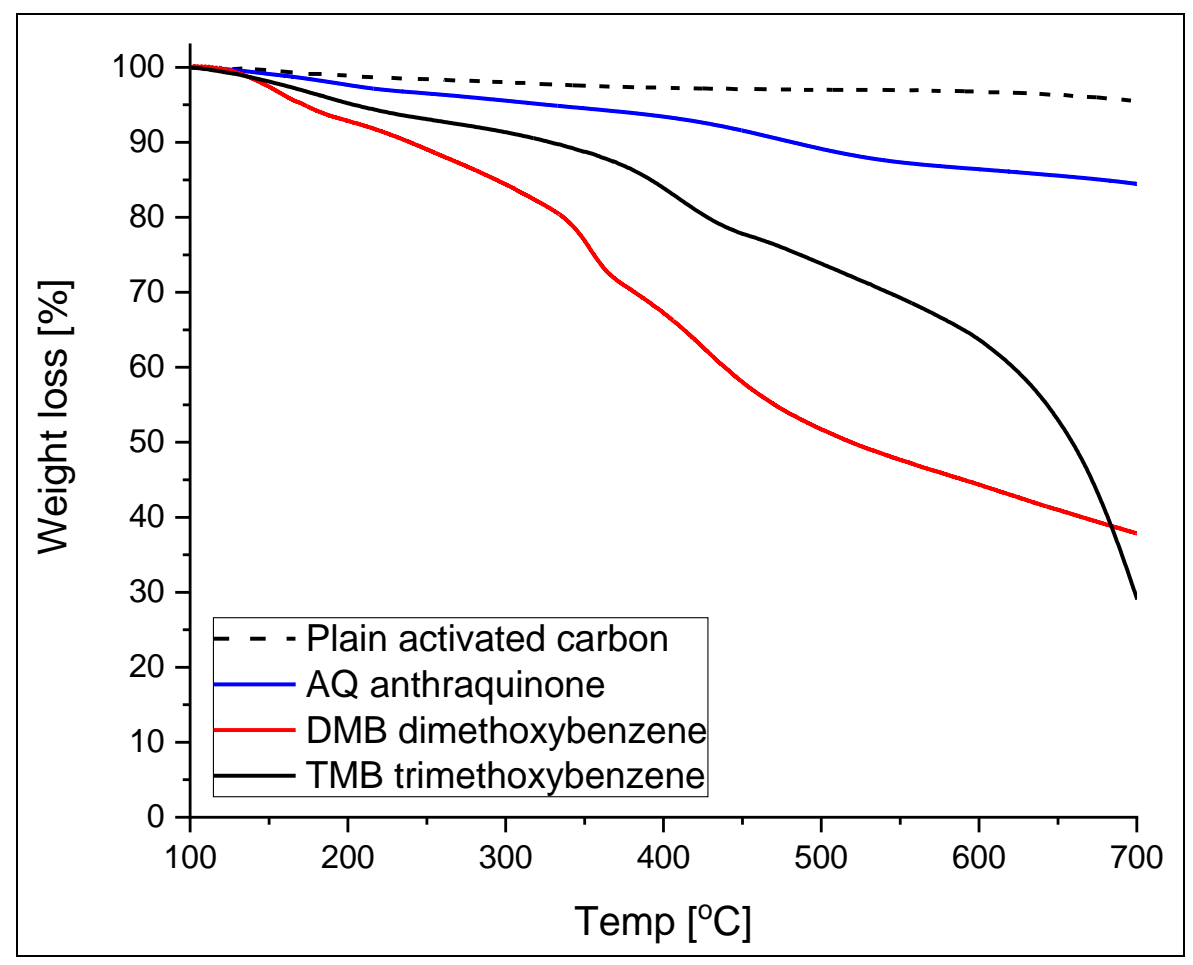

Figure 3: TGA performed under nitrogen at a temperature rate of 10K/min of grafted anthraquinone (blue), trimethoxybenzene (black) and dimethoxybenzene (red) vs. pristine activated carbon (dashed black).

X-ray photoelectron spectroscopy (XPS) may serve as powerful analytical tool in the case of surface modified electrodes. Using this technique, we can learn about the grafting products. In addition, XPS measurements may shed light on the electrochemical charging--discharging mechanism during positive and negative polarization. Unmodified and grafted TMA on carbon cloth was characterized by XPS. The survey spectra demonstrate $\mathrm{C} 1 \mathrm{~s}$ (at $286 \mathrm{eV}$ ), $\mathrm{O} 1 \mathrm{~s}$ (at $533 \mathrm{eV}$ ) and $\mathrm{N} 1 \mathrm{~s}$ (at $400 \mathrm{eV}$ ), evidence for the modification of the carbon surface.

\section{1s region}

The $C$ 1s spectra of the unmodified carbon (figure 4a) exhibits a main peak at $284.8 \mathrm{eV}$ and a manifold of unresolved peaks as a tail from $286 \mathrm{eV}$ to $292 \mathrm{eV}$ associated with higher electron deficient carbon centers. The grafted electrodes (figure 4d) exhibit a well resolved and strong peak at a binding energy (BE) of $286.5 \mathrm{eV}$. This peak may be assigned to the additional methoxy groups (oxidized carbon).

\section{1 s region}

Comparing the $\mathrm{O} 1 \mathrm{~s}$ spectra of modified (figure 4e) and unmodified carbon cloth (figure 4b) shows a difference in the size and location of the peaks. The grafted carbon cloth in the 01 s spectra shows considerably stronger peak than the unmodified. This is in agreement with the addition of 3 oxygens per grafted molecule. The intensification of the higher binding energy peak, at $533.5 \mathrm{eV}$ reflects enrichment in higher electron density oxygen sites. Meaning the oxygen is in higher oxidation state in the grafted electrodes. This result indicates that the oxygen originates from the carbons surface has lower levels of binding energy compare to the oxygen at the methoxy/hydroxy from the grafted molecule.

\section{N 1s region}

The N 1s core level spectra of the unmodified carbon cloth (figure 4c) all but a trace of detected nitrogen. The TMA grafted carbon cloth (figure $4 \mathrm{f}$ ) exhibits a strong peak of nitrogen at around $400 \mathrm{eV}$. A smaller, but still discernible peak, appears at slightly lower $\mathrm{BE}$, at ca. $402.2 \mathrm{eV}$. A reliable and unequivocal association of the peaks with specific $\mathrm{N}$-containing species is notoriously difficult. Nevertheless, the peak at ca. $400 \mathrm{eV}$ was suggested by others ${ }^{17,18}$ to be related to azo bridge ( $\mathrm{R}-\mathrm{N}=\mathrm{N}-\mathrm{R}$ ) of the grafted molecule 
to the carbon substrate surface. These originate from alternate grafting pathway using diazonium chemistry where the diazonium salt was directly coupled to the carbon substrate, instead of spontaneous degradation to $\mathrm{N}_{2}$ and aryl radical. In any case, even in case that indeed the strong peak is associated with the described side reaction, or other $\mathrm{N}$-containing moieties, they provide clear indication for the grafting process.

These results provide further evidence for the presence of TMA, verifying the success of the grafting.
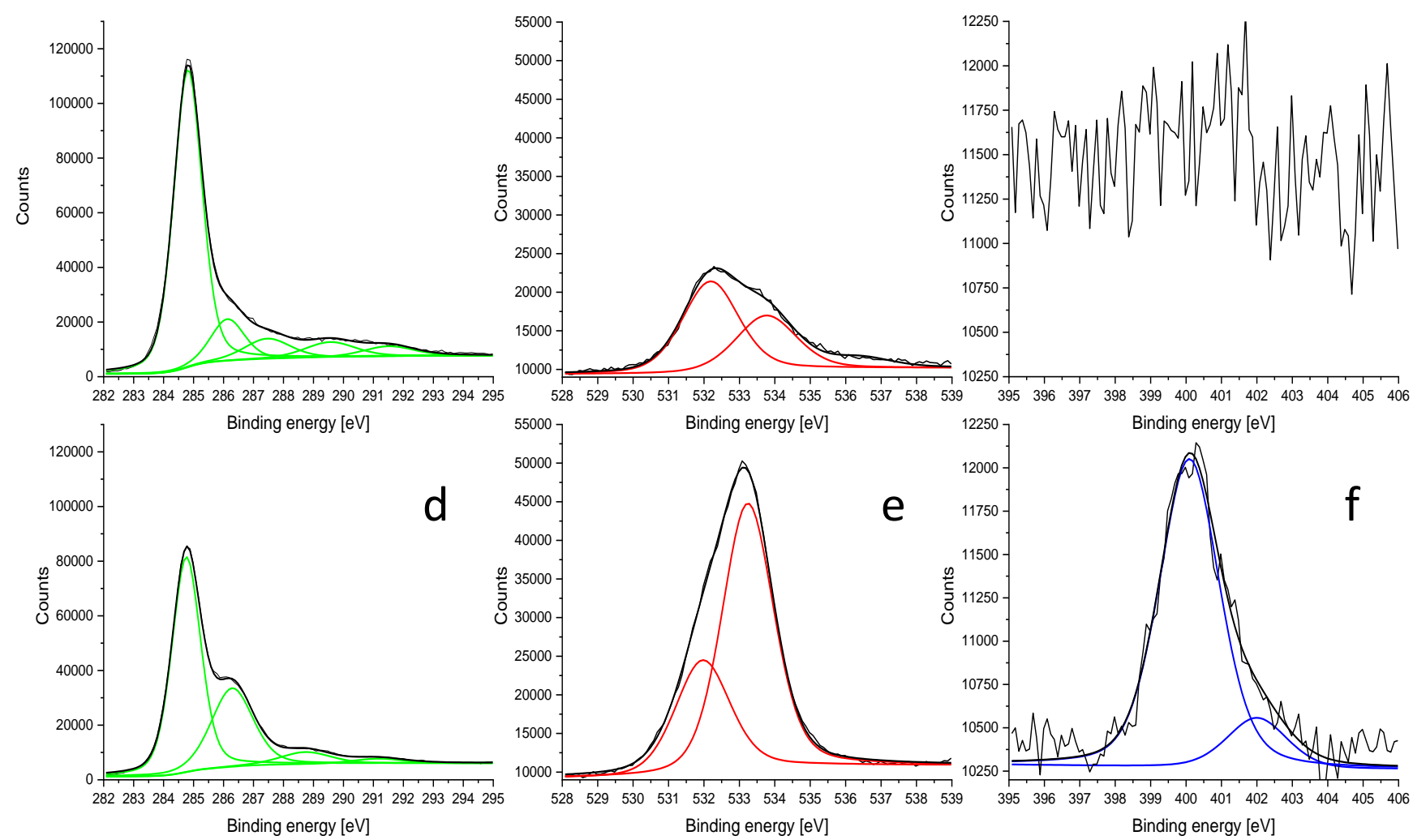

Figure 4: XPS spectra of unmodified carbon cloth $(a, b, c)$ and TMA modified carbon cloth $(d, e, f) C$ 1s (green), $O$ 1s (red) and $N$ 1s (blue) exhibit the profound changes due to the grafting process.

\begin{tabular}{|c|c|c|}
\hline Carbon cloth & Surface area $\left[\mathrm{m}^{2} / \mathrm{g}\right]$ & Surface area [\%] \\
\hline Pristine & $\sim 1500$ & 100 \\
\hline AQ modified & $\sim 500$ & 33 \\
\hline DMB modified & $\sim 350$ & 23 \\
\hline TMB modified & $\sim 140$ & 9 \\
\hline
\end{tabular}

Table 1: Surface area by BET of pristine and grafted carbon cloths in percentages compared to the initial specific surface area.

Brunauer-Emmett-Teller (BET) measurements summarized in table 1 show that grafting molecules to the surface of an activated carbon cloth is expected to be accompanied by a profound decrease in surface area with clear dependence on the nature of the grafted molecules. Figure 1 demonstrates the dynamic process of grafting and the formation of clusters depending on the grafted molecules. After grafting the first layer of molecules, AQ moieties have naked aromatic rings perpendicular to the surface of the carbon, which allows grafting of two molecules per initially grafted molecule in a second step, resulting in a rapid growth of grafted clusters on the carbon surface, until the process stops (either because the reagents are fully consumed, or termination by change of reaction's conditions). Here a yield of 20-25\% by weight due 
to additional $A Q$ molecules grafted to the carbon, is translates to a decrease in the surface area of the activated carbon by $66 \%$ - from about $1500 \mathrm{~m}^{2} / \mathrm{g}$ for the pristine activated carbon cloth to about $500 \mathrm{~m}^{2} / \mathrm{g}$ for the AQ-modified carbon cloth. Grafting the carbon cloth with DMB or TMB molecules enables a better coverage of its surface by these molecules, leading to a much drastic decrease in the specific surface area of the grafted carbon compared to the pristine activated carbon: from around $1500 \mathrm{~m}^{2} / \mathrm{g}$ to around 350 and $140 \mathrm{~m}^{2} / \mathrm{g}$ ( 23 and $9 \%$ of the pristine specific surface area), respectively. A similar blockage of pores was observed by Pognon et al. ${ }^{9}$. Most of the surface loss comes from blocking of micropore entrances by the grafted molecules, as typically observed with many carbon electrodes ${ }^{19}$.

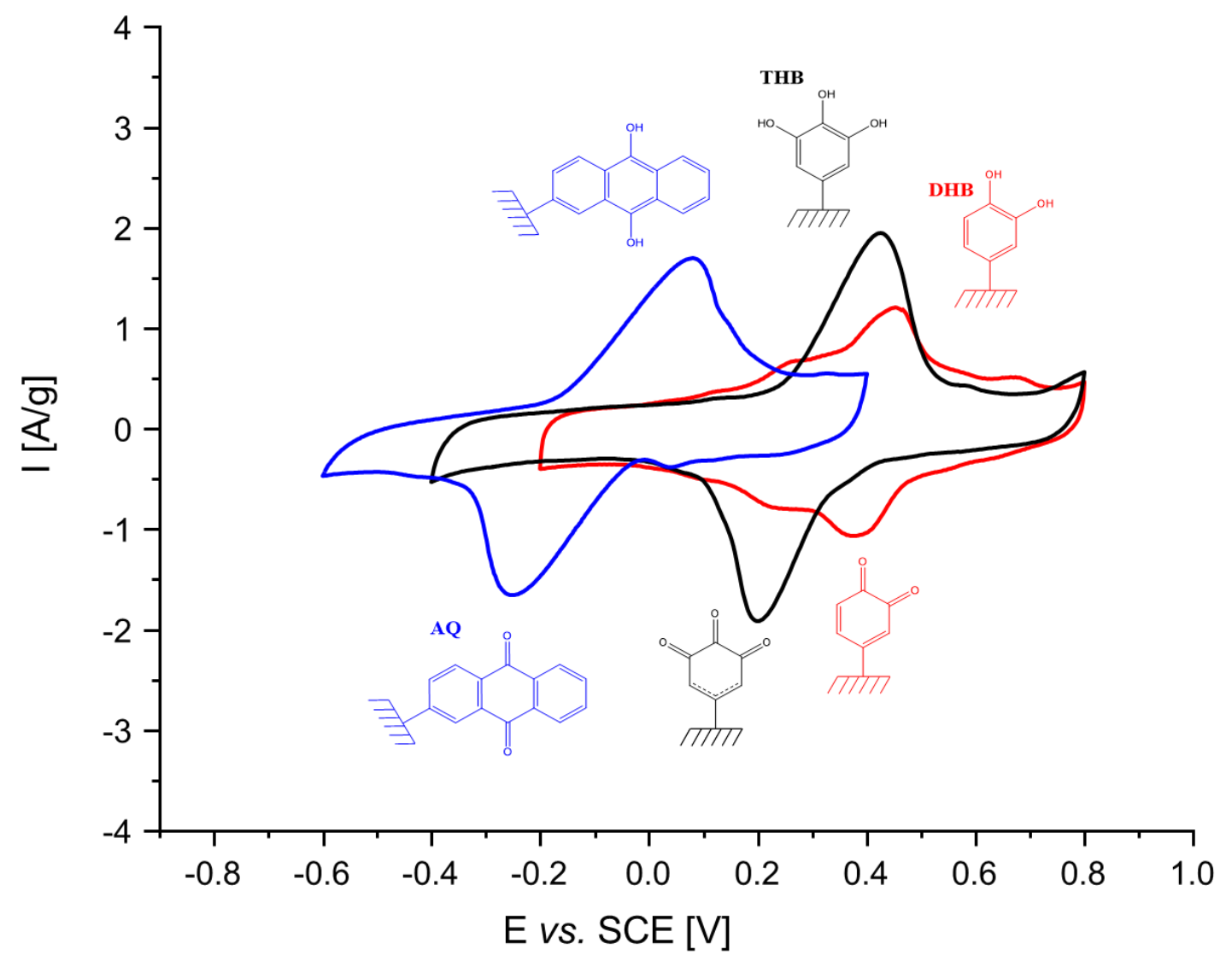

Figure 5: Cyclic voltammetry of grafted anthraquinone (blue), trihydroxybenzene (black) and dihydroxybenzene at a scan rate of $2 \mathrm{mV} / \mathrm{s}$ in $2 \mathrm{M} \mathrm{H}_{2} \mathrm{SO}_{4}$ electrolyte solution.

Cyclic voltammetric response of $\mathrm{AQ} / \mathrm{DHB} / \mathrm{THB}$ grafted $\mathrm{Kynol}^{\mathrm{TM}}$ carbon cloth electrodes is shown in figure 5 , which compares relevant steady-state voltammograms of the three electrodes on a single chart. It can be clearly observed that the electrodes containing the hydroxybenzene derivatives exhibit redox activity at $0.4 \mathrm{~V}$ above that of anthraquinone. Since the experiments were carried out under the same conditions (cell assembly, electrolyte solution, mass loading and scan rate), the results reflect the different levels of loading, deprotection, and redox potential. The different locations of the redox peaks are related to the molecules electronic structure, namely, their molecular structure and ionization energy gap. The hydroquinone moiety in $A Q$ is stabilized by two aromatic rings, allowing reduction-oxidation at lower potentials relative to the hydroxybenzene species. DHB shows two asymmetric peaks, reflecting the different hydroxy moieties, while THB shows a single sharp peak corresponding to the symmetrical structure and subsequent ionization steps.

The grafted molecule is the protected 3,4,5-trimethoxyaniline. This methoxy derivative is inactive electrochemically and requires activation/deprotection. Usually, most of the deprotection to the active hydroxy 
specie ${ }^{14}$ is done by reacting the aromatic methoxy with $\mathrm{BBr}_{3}$ before the grafting process. An in situ cleavage of methoxy to hydroxy by electrochemical means (at $0.7 \mathrm{~V} v$ s. SCE in $2 \mathrm{M}$ sulfuric acid) is suggested in figure 6. These conditions promote irreversible reactions that transform the aromatic methoxy to hydroxy species as described by Cougnon et $a .^{20}$, and is illustrated in figure 6. Polarization of the electrodes up to $0.6 \mathrm{~V}$ vs. SCE activate them. Early protonation of the oxygen directs an E1 reaction of the sulfate ions on the positively charged methyl. This process is demonstrated well in the cyclic voltammetry of electrodes containing THB grafted on Kynol ${ }^{\mathrm{TM}}$ in figure 7 (left, $1^{\text {st }} \mathrm{cycle}$ ). The peak current related to the methoxy cleavage appears at $0.6-0.7 \mathrm{~V}$ and it reflects the transformation of the grafted protected TMB moieties to the reversibly redox active (grafted) THB species $0.1-0.5 \mathrm{~V}$. cycling starts at $0.4 \mathrm{~V}$ vs. SCE towards reduction, the first reduction peak at $0.2 \mathrm{~V} v$ s. SCE is the reversible peak of hydroxy benzene that was already partialy deprotected by the cell conditioning $\left(2 \mathrm{M} \mathrm{H}_{2} \mathrm{SO}_{4}\right.$ and $\mathrm{OCV}$ of $0.4 \mathrm{~V}$ vs. SCE), the second reduction peak at $-0.2 \mathrm{~V} v$ s. SCE is ireversible peak that can be adsorbed diazo derivative trapped in the porosity of the carbon, reduced and grafted to the surface.

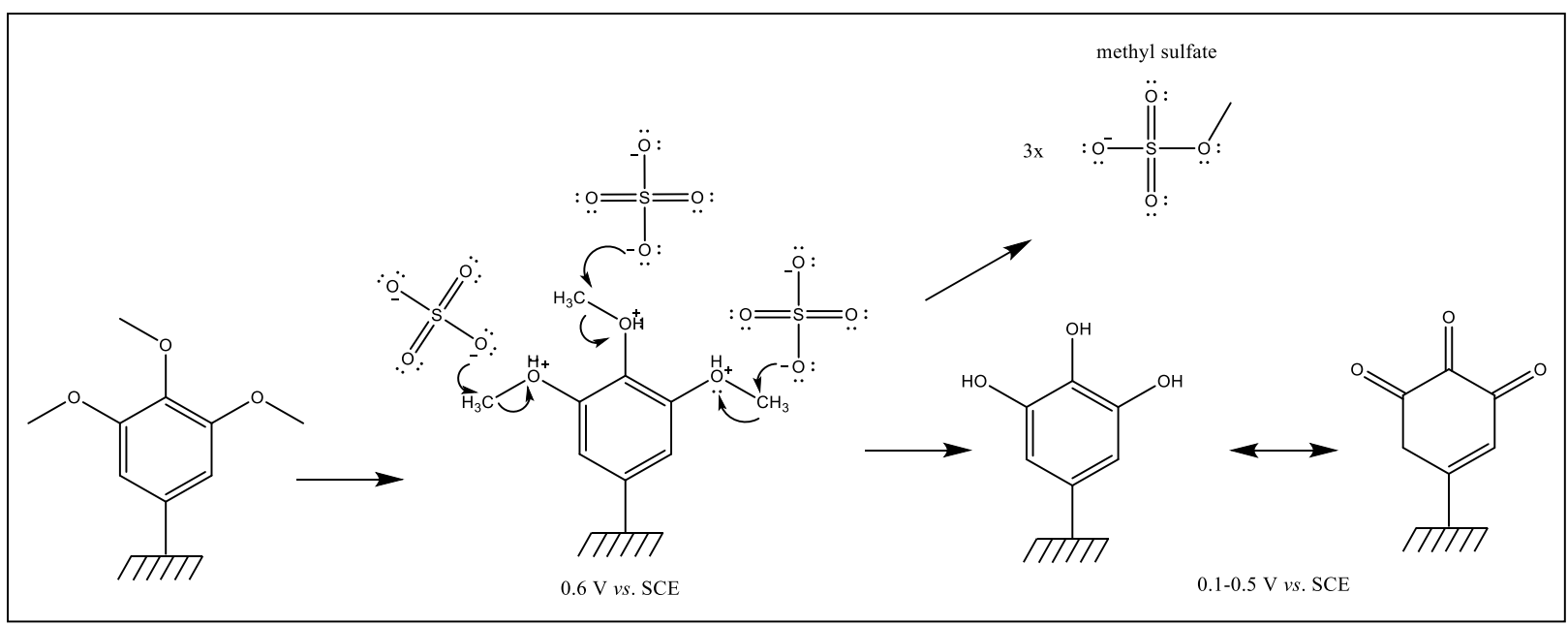

Figure 6: In situ electrochemical deprotection of grafted trimethoxybenzene to the redox active (grafted) trihydroxybenzene. Early protonation of the methoxy oxygen directs an E1 reaction of the poor sulfate ion nucleophile to the positively charged methyl, with methyl sulfate leaving group.

Figure 7 reflects the electrochemically irreversible formation of the grafted hydroxy moiety from the methoxy derivative at $2 \mathrm{mV} / \mathrm{s}$ (left panel) and at different scan rates (right panel).
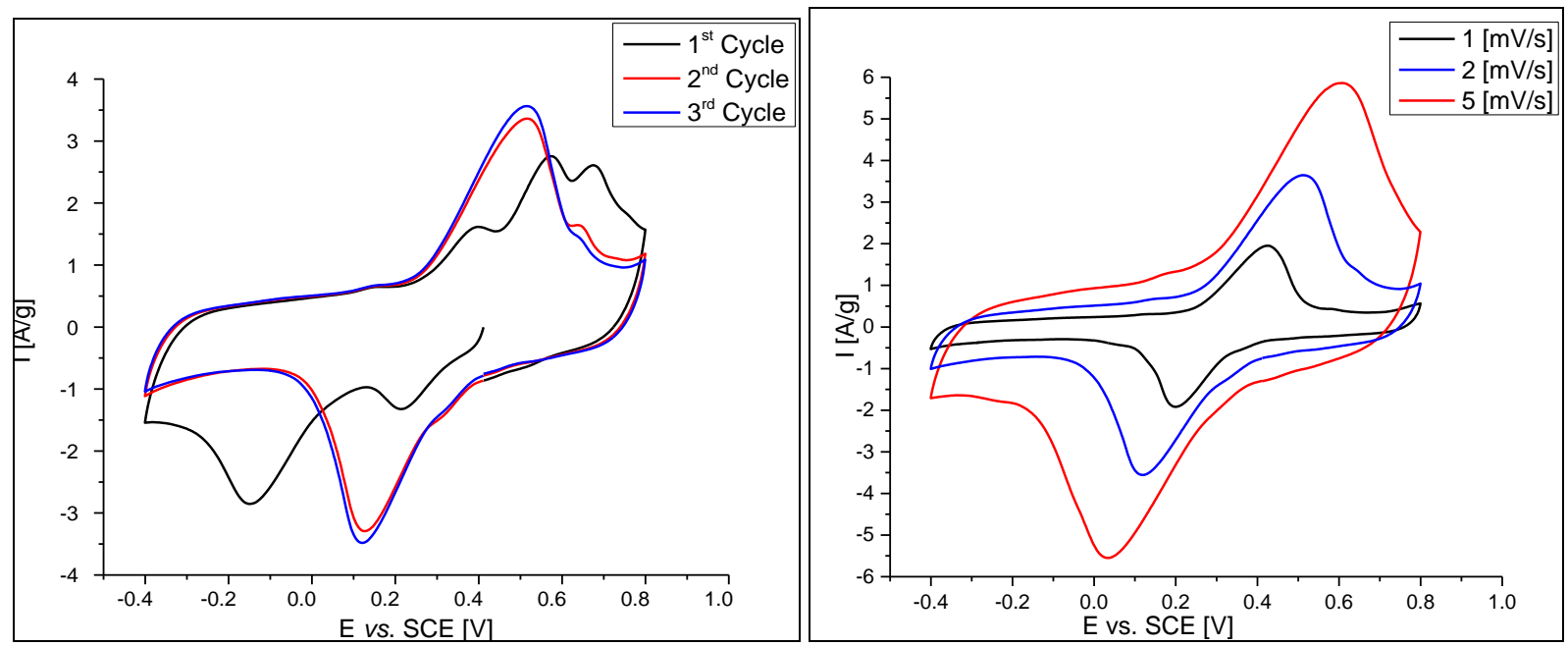

Figure 7: The first voltammetric cycles of electrodes comprising trimethoxybenzene grafted on carbon cloth, showing the cleavage of trimethoxy- to trihydroxybenzene up to the third cycle at a scan rate of $2 \mathrm{mV} / \mathrm{s}$ (left) and steady state voltammograms at different scan rates (right) in $2 \mathrm{M} \mathrm{H}_{2} \mathrm{SO}_{4}$ electrolyte solution. 

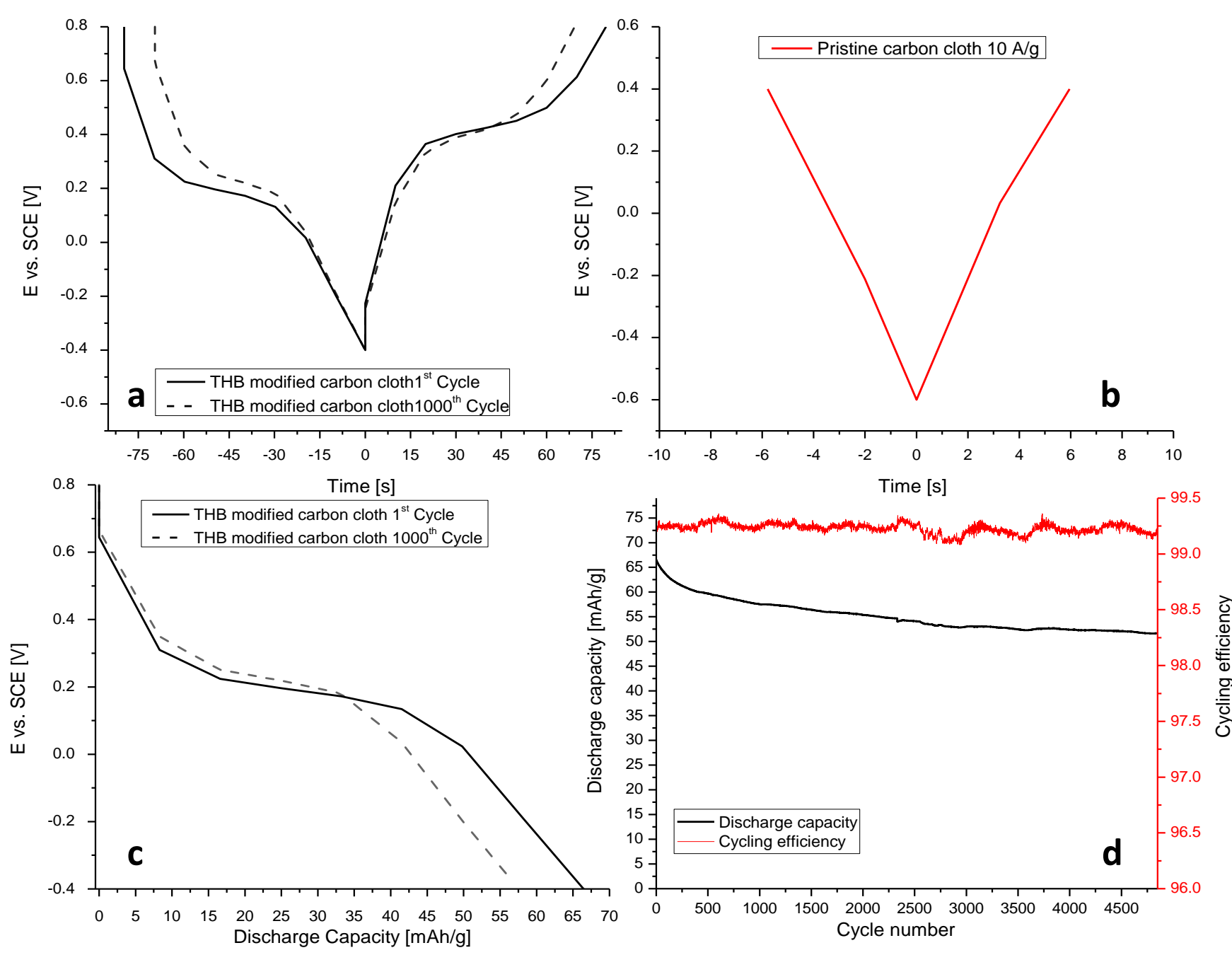

Figure 8: Galvanostatic charge/discharge in $2 \mathrm{M} \mathrm{H}_{2} \mathrm{SO}_{4}$ showing the voltage profiles of the $1^{\text {st }}$ and $1000^{\text {th }}$ cycle of THB modified carbon cloth at $3 \mathrm{~A} / \mathrm{g}(\mathrm{a})$ and pristine carbon cloth at $10 \mathrm{~A} / \mathrm{g}(\mathrm{b})$, voltage vs. capacity (c), and discharge capacity and cycling efficiency for prolonged cycling (d).

Figure 8a shows typical galvanostatic voltage profiles of electrodes comprising $\mathrm{Kynol}^{\mathrm{TM}}$ carbon cloth on which THB molecules were grafted as explained and described above, comparing the first and $1000^{\text {th }}$ cycles. Figure $8 \mathrm{~b}$ shows for comparison a typical galvanostatic voltage profile of an electrode comprising pristine $\mathrm{Kynol}^{\mathrm{TM}}$ carbon cloth. The difference between charts $8 \mathrm{a}$ and $8 \mathrm{~b}$ is striking; the latter reflects a purely capacitive behavior of the pristine carbon electrode, while the former reflects a typical redox (battery type) behavior, showing a clear plateau at around $0.1-0.5 \mathrm{~V}$ vs. SCE, corresponding to a faradaic reaction. Moreover, this comparison shows how the effective grafting changes the electrochemical window of the modified carbon electrodes, compared to the pristine activated carbon electrode (see the voltage scales in the two charts). Figure $8 \mathrm{c}$ compares the first and $1000^{\text {th }}$ galvanostatic discharge voltage profiles of the electrode THB-modified Kyno ${ }^{\mathrm{TM}}$ carbon cloth in terms of specific capacity, which can reach values $>65 \mathrm{mAh} / \mathrm{g}$. This value means a gain of more than $50 \%$ in specific capacity due to grafting with THB compared to the pristine electrode (with maximal specific capacity at low rates $>40 \mathrm{mAh} / \mathrm{g}$ ), although grafting reduces the specific surface area by around $90 \%$ (see Table 1 ).

Figure $8 \mathrm{~d}$ provides typical results of prolonged galvanostatic charge-discharge cycling measurements and prolonged over 4500 cycles with THB grafted $\mathrm{Kynol}^{\mathrm{TM}}$ cloth electrodes (a plot of specific capacity vs. cycle number) at a current density of $3 \mathrm{~A} / \mathrm{g}$ in $2 \mathrm{M} \mathrm{H}_{2} \mathrm{SO}_{4}$ electrolyte solution. As can be seen, these electrodes provide discharge capacity starting from $65 \mathrm{mAh} / \mathrm{g}$ that degrades to a stable $55 \mathrm{mAh} / \mathrm{g}$ after about 2000 
cycles. AQ and DHB modified carbon fabric electrodes were also reported ${ }^{14,16}$ as showing a good gain in specific capacity due to grafting. For standard activated carbon electrodes, a very good cycling ability was reported in $\mathrm{H}_{2} \mathrm{SO}_{4}$, but with only a low amount of grafted $\mathrm{AQ}$ molecules ${ }^{4}$. Recently ${ }^{8,15}$, good stability during prolonged cycling was also demonstrated for electrodes comprising activated carbon cloth grafted with $\mathrm{AQ}$ or DHB molecules. Table 2 summarizes results from the present study with previous studies with other types of electrodes. This comparison is only qualitative since the conditions of the cycling experiments in the previous studies were not quite similar to those related to the present work.

\begin{tabular}{|c|c|c|c|c|c|}
\hline $\begin{array}{c}\text { Grafted } \\
\text { moleculeCarbon } \\
\text { cloth }\end{array}$ & $\begin{array}{l}\text { Electrolyte } \\
\text { solution }\end{array}$ & $\begin{array}{c}\text { Mass } \\
\text { loading [\%] }\end{array}$ & $\begin{array}{l}\text { Potential } \\
\text { vs. SCE [V] }\end{array}$ & $\begin{array}{l}\text { Capacity } \\
\text { [mAh/g] }\end{array}$ & $\begin{array}{c}\text { Current } \\
\text { density }[A / g]\end{array}$ \\
\hline Pristine cloth ${ }^{8}$ & $6 \mathrm{M} \mathrm{KOH}$ & 0 & $-0.4--1.4$ & 25 & 10 \\
\hline Pristine cloth ${ }^{8}$ & $1 \mathrm{M} \mathrm{H}_{2} \mathrm{SO}_{4}$ & 0 & $0.4--0.6$ & 20 & 10 \\
\hline AQ modified $^{8}$ & $6 \mathrm{M} \mathrm{KOH}$ & 25 & $-0.4--1.4$ & 55 & 10 \\
\hline AQ modified $^{8}$ & $1 \mathrm{M} \mathrm{H}_{2} \mathrm{SO}_{4}$ & 25 & $0.4--0.6$ & 65 & 10 \\
\hline DHB modified ${ }^{15}$ & $1 \mathrm{M} \mathrm{H}_{2} \mathrm{SO}_{4}$ & 41 & $0.8--0.2$ & 75 & 1 \\
\hline THB modified & $2 \mathrm{M} \mathrm{H}_{2} \mathrm{SO}_{4}$ & 25 & $0.8--0.4$ & 65 & 3 \\
\hline
\end{tabular}

Table 2: Comparison between the performance of pristine carbon cloth electrodes and electrodes comprising Kynol ${ }^{T M}$ 507-20 carbon cloth modified (by grafting) with $A Q, D H B$ and $T H B$.

The theoretical capacity of THB modified carbon cloth electrodes, considering the number of molecules grafted and the total number of available redox centers, is $160 \mathrm{mAh} / \mathrm{g}$, while only about $40 \%$ of this capacity was obtained. This can be explained in two ways. Methoxy deprotection, which requires an early protonation triggered at $0.7 \mathrm{~V}$ vs. SCE. This may lead to a scarcity of protons near the electrode due to the opposite flux of ions. The second one is insufficient conditions for the redox reaction: extracting three electrons from THB to form oxidized tri-keto derivative may interfere with charge dislocation by two vicinal oxygens sharing a proton, leading to only partial electron transfer. The aim here was to demonstrate, to the best of our knowledge for the first time, the potential of grafting activated carbon electrodes by THB, and calls in fact for a further optimization work. Other aspects such as rate capability, temperature dependence, the effect of electrolyte solutions composition should be studied with optimized electrodes which exhibit the maximal specific capacity that can be extracted from modifying carbon electrodes with THB.

\section{Conclusion}

A new grafted molecule is presented with significant advantages for enhancing the specific capacity of activated carbon electrodes. The modification by grafting realizes pseudocapacitive electrodes that narrow the specific capacity gap between batteries and supercapacitors electrodes. 3,4,5 trihydroxybenzene (THB) exhibits three electrons available for redox reactions, possessing a theoretical capacity of $638 \mathrm{mAh} / \mathrm{g}$. Unavailability of sites for regrafting means that only monolayers of THB can be deposited across the surface of the carbon.

The obtained grafted carbon was characterized physically and electrochemically. As observed in cyclic voltammetry and galvanostatic charge discharge experiments, the modified carbon cloth electrodes demonstrate a clear faradaic contribution on top of the natural double layer capacitance under acidic conditions. Specific capacities start from $65 \mathrm{mAh} / \mathrm{g}$ and slowly degrade after the first 2000 cycles to a stable capacity of around $55 \mathrm{mAh} / \mathrm{g}$, which persisted for over 2000 additional cycles. The theoretical capacity for $25 \%$ mass loading by THB is $160 \mathrm{mAh} / \mathrm{g}$. Practically, only about $40 \%$ of this capacity was available, probably mainly due to incomplete methoxy deprotection. The results obtained herein definitely encourage an interesting future work (beyond the scope of this paper, which intention was to present this area) aiming at finding a way to quantify the extent of de-protection and to determine if the 
limited capacity of TMB (compared with theoretical) comes from the presence of centers that are inactive or because the centers are unable to exchange all 3 electrons. It is important to determine the level of demethylation and if we succeed on extracting 3 electrons from each grafted molecule or each redox center. For carrying out properly such a study, it is mandatory to use flat substrate electrodes as a starting point for a rigorous analytical work. Such a future work is underway.

\section{Acknowledgment}

Partial support for this work was obtained from the Israel Ministry of Energy and National research center for electrochemical propulsion (INREP) project supported by the Prime Minister Office and the Committee for High Education.

\section{References}

1. P. Simon, Y. Gogotsi, and B. Dunn, Science (80-. )., 343, 1210-1211 (2014).

2. M. Winter and R. J. Brodd, Chem. Rev., 104, 4245-4269 (2004).

3. V. Etacheri, R. Marom, R. Elazari, G. Salitra, and D. Aurbach, Energy Environ. Sci., 4, 3243-3262 (2011).

4. G. Pognon, T. Brousse, L. Demarconnay, and D. Bélanger, J. Power Sources, 196, 4117-4122 (2011).

5. B. D. Assresahegn, T. Brousse, and D. Bélanger, Carbon N. Y., 92, 362-381 (2015).

6. C. P. Galloway,. US Pat., 5,851,280, 1998; Cabot Corporation, US. Pat., 5,851,280, 1998.

7. J. A. Belmont., US Pat., 5,554,739, 1996; Cabot Corporation, US. Pat., 5,554,739, 1996.

8. D. Malka et al., J. Electrochem. Soc., 165, A3342-A3349 (2018).

9. G. Pognon, T. Brousse, and D. Bélanger, Carbon N. Y., 49, 1340-1348 (2011).

10. A. Le Comte, G. Pognon, T. Brousse, and D. Bélanger, Electrochemistry, 81, 863-866 (2013).

11. A. Le Comte, T. Brousse, and D. Bélanger, Electrochim. Acta, 137, 447-453 (2014).

12. K. Kalinathan, D. P. DesRoches, X. Liu, and P. G. Pickup, J. Power Sources, 181, 182-185 (2008).

13. E. Lebègue, T. Brousse, J. Gaubicher, and C. Cougnon, Electrochem. commun., 34, 14-17 (2013).

14. G. Pognon, C. Cougnon, D. Mayilukila, and D. Bélanger, ACS Appl. Mater. Interfaces, 4, 3788-3796 (2012).

15. D. Malka et al., J. Electrochem. Soc., 166, A1147-A1153 (2019).

16. Z. Algharaibeh and P. G. Pickup, Electrochem. commun., 13, 147-149 (2011).

17. A. Le Comte, T. Brousse, and D. Bélanger, Electrochim. Acta, 197, 139-145 (2016).

18. M. Toupin and D. Bélanger, J. Phys. Chem. C, 111, 5394-5401 (2007).

19. K. Brousse et al., Electrochim. Acta, 246, 391-398 (2017).

20. C. Cougnon, E. Lebègue, and G. Pognon, J. Power Sources, 274, 551-559 (2015). 